\title{
Epigenetic silencing of KLF2 by long non- coding RNA SNHG1 inhibits periodontal ligament stem cell osteogenesis differentiation
}

\author{
Zhaobao Li, Xiangjun Guo and Shuainan Wu (D)
}

\begin{abstract}
Background: Exploring the effects of IncRNA SNHG1 in the process of osteogenic differentiation of periodontal ligament stem cells (PDLSCS) would provide novel therapeutic strategies for tissue regeneration.

Methods: Loss-of-function and gain-of-function assays were induced by lentivirus. The osteogenic differentiation of PDLSCs were assessed by ALP staining and Alizarin Red staining as well as the mRNA and protein levels of osteogenic marker genes osterix, osteocalcin, and alkaline phosphatase through qRT-PCR and western blot. RNA immunoprecipitation assay and chromatin immunoprecipitation assays were performed to uncover the interaction between SNHG1 and EZH2.

Results: Our analysis revealed that SNHG1 was downregulated and KLF2 was upregulated during the osteogenic induction differentiation of PDLSCs. SNHG1 inhibited while KLF2 promoted osteogenic differentiation of PDLSCs. SNHG1 directly interact with the histone methyltransferase enhancer of the zeste homolog 2 (EZH2) and modulate the histone methylation of promoter of Kruppel-like factor 2 (KLF2) and altered the progress osteogenic differentiation of PDLSCS.

Conclusions: Taken together, SNHG1 inhibited the osteogenic differentiation of PDLSCs through EZH2-mediated H3K27me3 methylation of KLF2 promotor and provided a novel class of therapeutic targets for regenerate dental tissues.
\end{abstract}

Keywords: SNHG1, EZH2, KLF2, PDLSCs, Osteogenic differentiation

\section{Background}

Human periodontal mesenchymal stem cells (PDLSCs) are a group of mesenchymal stem cells which are isolated from periodontal ligament tissue with multiple differentiation capability and show a promising potential of being used to regenerate supporting tissues for the patients [1-3]. Periodontitis is a highly prevalent chronic inflammatory bone disease and could destroy the

\footnotetext{
* Correspondence: Wshn1986@126.com

Department of Stomatology Clinic, Cangzhou Central Hospital, Cangzhou 061000, Hebei, China
}

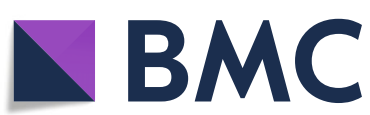

(c) The Author(s). 2020 Open Access This article is licensed under a Creative Commons Attribution 4.0 International License, which permits use, sharing, adaptation, distribution and reproduction in any medium or format, as long as you give appropriate credit to the original author(s) and the source, provide a link to the Creative Commons licence, and indicate if changes were made. The images or other third party material in this article are included in the article's Creative Commons licence, unless indicated otherwise in a credit line to the material. If material is not included in the article's Creative Commons licence and your intended use is not permitted by statutory regulation or exceeds the permitted use, you will need to obtain permission directly from the copyright holder. To view a copy of this licence, visit http://creativecommons.org/licenses/by/4.0/. The Creative Commons Public Domain Dedication waiver (http://creativecommons.org/publicdomain/zero/1.0/) applies to the data made available in this article, unless otherwise stated in a credit line to the data.

periodontal structures, including alveolar bone, periodontal ligament, and root cementum, which eventually lead to tooth-loss [4]. Therefore, better understanding of the mechanism that governs PDLSCs osteogenic differentiation would greatly be beneficial to the development of novel therapeutic strategies for tissue regeneration.

Long noncoding RNAs (lncRNAs), with longer than 200 nucleotides in length, have recently been noticed as the function on malignant processes, such as carcinogenesis [5-7] and periodontitis [8-10]. LncRNA ANCR was report to inhibit osteogenic differentiation of PDLS 
Cs via sponging miRNA-758 [11]. Similarly, lncRNA H19 also was reported to promote osteogenic differentiation of rat ectomesenchymal stem cells through enhancing the expressions of osteogenic markers, beta-catenin, and target genes of Wnt/beta-catenin signaling pathway partly through sponging miR-22 and miR-141 [12]. LncRNA SNHG1 could inhibit osteogenic differentiation of bone marrow mesenchymal stem cells through p38 MAPK pathway [13]. In brief, lncRNA plays an important role in osteogenic differentiation of PDLSCs and our study will further investigate the effects of lncRNA SNHG1 in osteogenic differentiation of PDLSCs and explore the underlying mechanism.

LncRNAs are reported to exert their functions through different molecular mechanisms and impact the transcription of downstream factors by regulating histone acetylation or methylation as well as DNA methylation or hydroxy methylation [14-16]. EZH2 (enhancer of the zeste homolog 2) which was characterized as a histone methylase was also found to interact with lncRNAs and then impact the transcription of downstream factors [17]. For instance, LINC00114 suppressed miR-133b expression via EZH2/DNMT1-mediated methylation of its promoter region and facilitated colorectal cancer development [16]. Long noncoding RNA NEAT1 (nuclear-enriched abundant transcript 1) inhibited hepatocyte proliferation in fulminant hepatic failure via strengthened recruitment of EZH2 to the LATS2 (large tumor-suppressor kinase 2) promoter region [18]. Importantly, SNHG1 was also proved to interact with EZH2 and inhibit CDKN2B (cyclin-dependent kinase inhibitor 2B) and CDKN1A (cyclin-dependent kinase inhibitor 1A) transcription $[19,20]$. However, the function of SNHG1 and EZH2 in osteogenic differentiation of PDLSCs was still less reported.

The purpose of the current study was to investigate whether lncRNA SNHG1 was mechanistically involved in the osteogenic differentiation of PDLSCs. Our results identified that SNHG1 was a regulator of PDLSC osteogenesis and found SNHG1 participates in epigenetic repression of KLF2 by interacting with EZH2 in the progress of the osteogenic differentiation of PDLSCs. Our results provided novel insights into the mechanism that underlies the osteogenic differentiation of PDLSCs and could serve as novel therapeutic strategies for regenerate dental tissues.

\section{Materials and methods}

\section{Isolation and culture of primary cells}

The first or the second premolar teeth from 10 patients without periodontal disease (age 18-25 years) were respectively collected for orthodontic purposes. Ten premolar teeth were pooled and used to collect the periodontal membrane tissue. This study was approved by the ethics committee of Cangzhou Central Hospital. Written informed consent was obtained from all participants. All research involving human stem cells complied with the ISSCR "Guidelines for the Conduct of Human Embryonic Stem Cell Research."

PDLSCs were isolated, cultured, and identified as previously reported [21]. Briefly, periodontal ligament tissues were then separated from middle $1 / 3$ of teeth roots. Tissue fragments were then digested with collagenase type I and dispase (Sigma-Aldrich) for $1 \mathrm{~h}$ at $37^{\circ} \mathrm{C}$. Cell suspensions were then filtered through a $70-\mu \mathrm{m}$ cell strainer (BD Biosciences) and single cell suspension were maintained in complete $\alpha$-MEM (HyClone) with $10 \%$ FBS (Invitrogen) at $37^{\circ} \mathrm{C}$ with $5 \% \mathrm{CO}_{2}$. The culture medium was changed every 3 days. Cells at passages $3-5$ were used in subsequent experiments.

\section{Flow cytometry analysis}

For immunophenotype characterization, $1 \times 10^{5}$ PDLSCs were incubated with anti-human stem cell surfacelabeled antibodies including CD73-phycoerythrin and CD90-phycoerythrin (BD Biosciences, USA), antihematopoietic marker CD34 antibodies (BD Biosciences, USA), and anti-endothelial cell marker CD31 antibodies (BD Biosciences, USA). All flow cytometry tests were performed on a FACSAria (BD Bioscience).

\section{Multiple differentiation of cells in vitro}

The multiple differentiation capacities of PDLSCs were determined according to the methods previously described $[22,23]$. Briefly, PDLSCs were cultured into a 6-well plate in a density of $1 \times 10^{6}$ cell per well without inducers until $80 \%$ confluence. For osteogenic induction, osteogenic differentiation induction medium $(50 \mu \mathrm{g} / \mathrm{mL}$ of ascorbic acid (Sigma), $10 \mathrm{mM}$ of betaglycerophosphate (Sigma), and $10 \mathrm{nM}$ of dexamethasone (Sigma) diluted in 10\% FBS a-MEM) was given for 2 weeks and PDLSCs were identified with alizarin red and ALP staining. For adipogenic differentiation, adipogenic medium (StemPro Adipogenesis Differentiation Kit) for 14 days and then fixed and stained with oil red $\mathrm{O}$ staining. For chondrogenic differentiation, $1 \times 10^{5}$ of cells were cultured in a 24-well cell culture dish with the chondrogenic medium (StemPro Chondrogenesis Differentiation Kit). After 2 weeks of induction, the clusters were fixed with $4 \%$ paraformaldehyde, embedded in paraffin. Five-micrometer section was used for alcian blue staining (Cyagen, HUXMA-90041) for identification.

\section{Virus infection}

Virus for SNHG1, KLF2, and EZH2 overexpression and the specific shRNA for KLF2, SNHG1, and EZH2 were prepared by GenePharma. Viral infection was conducted as Liu et al. described [24]. PDLSCs were inoculated 
overnight and infected with lentiviruses in the presence of polybrene $(6 \mu \mathrm{g} / \mathrm{mL}$; Sigma-Aldrich) for $6 \mathrm{~h}$. Cells were then selected with puromycin treatment for $48 \mathrm{~h}$. Resistant clones were collected and was identified via real-time RT-PCR and western blot. The sequences of shRNA are shown in supplementary Table 1.

\section{Alkaline phosphatase (ALP) activity and ALP staining assay}

PDLSCs were seeded into 96-well plates with a density of $1 \times 10^{5}$ cells/well. Fourteen days after induction, ALP activity was detected through the ALP assay kit (Jiancheng Technology Co.). ALP activity was determined at $405 \mathrm{~nm}$ using p-nitro-phenyl phosphate as a substrate. Cellular ALP was also visualized by using Alkaline Phosphatase Color Development Kit (Beyotime) according to the manufacturer's protocol.

\section{Alizarin red staining and quantification} PDLSCs were transferred into 24-well plates with a density of $2 \times 10^{5}$ cells/well. Fourteen days after induction, alizarin red staining measurement was performed by using Alizarin Red S $(0.2 \%$, Solarbio, $\mathrm{pH}=8.3)$ at $24-$ $26^{\circ} \mathrm{C}$. After washing in PBS, the matrix mineralization level was observed using an inverted microscope. To detect the concentration of calcium deposits, the Alizarin Red dye in the PDLSCs was extracted with $400 \mu$ of $10 \%$ $(\mathrm{w} / \mathrm{v})$ cetylpyridinium chloride in $10 \mathrm{mM}$ sodium phosphate solution for $10 \mathrm{~min}$ and then quantified on a UV-Vis spectrometer at $562 \mathrm{~nm}$.

\section{Real-time quantitative polymerase chain reaction}

Total RNAs were extract using TRIzol reagent (CW Biotech) followed the manufacturers' instructions. Super cDNA First-Strand Synthesis Kit (CW Biotech) and SYBR Green Real-Time PCR Master Mix (Invitrogen) were used for mRNA detection. GAPDH was used as endogenous controls for mRNA detection and the relative level of gene expression was calculated based on the $2^{- \text {DDCq }}$ method. The primers are shown in supplementary Table 2 .

\section{Western blot analysis}

PDLSCs were lysed in RIPA buffer (Sigma-Aldrich) and western blotting was performed using SDS-PAGE and then transferred to PVDF membranes (Merck Millipore). After blocking in 5\% milk, the blot was incubated with primary and secondary antibodies. All antibodies were purchased from Abcam. The enhanced chemiluminescence (ECL) reagents were added to visualize the protein band and GAPDH was taken as the loading control. The gray intensity analysis was performed using Image Pro Plus (Media Cybernetics). All antibodies were purchased form Abcam and listed in supplementary Table 3.
RNA immunoprecipitation (RIP)-PCR assay

RIP was performed using the RIP Kit (BersinBio) with EZH2 antibody according to the manufacturer's instructions. $1 \times 10^{7}$ treated PDLSCs were lysed with RIP buffer. The cell lysate was then incubated with sepharose beads (Bio-Rad, Hercules, CA, USA) pre-coated with EZH2 antibody (Abcam). Immunoglobulin $G$ antibody served as the control in this study. qRT-PCR was performed as described above to measure the levels of SNHG1 associated with EZH2. The results were normalized relative to the input control.

\section{Chromatin immunoprecipitation (ChIP)-PCR assay}

ChIP was carried out using the Pierce Agarose ChIP kit (ThermoFisher Scientific) as described previously [20]. Briefly, $1 \times 10^{7}$ treated PDLSCs were cross-linked with $1 \%$ formaldehyde solution for $10 \mathrm{~min}$ at room temperature. DNA fragments ranging from 200 to 500 bp were generated via sonication. Then, the lysates were immunoprecipitated with anti-EZH2, anti-H3K27me3, or normal rabbit IgG antibody. Immunoprecipitated DNAs were analyzed by qRT-PCR. The results were normalized relative to the input control. All antibodies were purchased form Abcam and listed in supplementary Table 3.

\section{Statistical analysis}

The results were presented as means \pm standard deviation using Graphpad Prism 6 (GraphPad Software Inc.). All the data used in study normally distributed and meet homogeneity test for variance. The collected data for comparisons were analyzed by $\mathrm{T}$ Student's $t$ test and one-way analysis of variance (ANOVA) for comparisons. $p<0.05$ was considered statistically significant.

\section{Results}

SNHG1 and KLF2 expression in PDLSCs during the osteogenic differentiation

PDLSCs were successfully isolated and exhibited the positive mesenchymal stem cell surface markers, including CD73 and CD90, whereas the negative hematopoietic marker CD34 and endothelial cell marker CD31 (Fig. 1A). PDLSCs also were found to differentiate into osteogenic, chondrogenic, and adipogenic lineages after relevant induction protocols (Fig. 1B). To investigate whether SNHG1 and KLF2 are involved in regulating osteogenic differentiation, PDLSCs were cultured in osteogenic differentiation medium (OM). The activity of osteoblastic marker ALP was significantly increased after OM treatment for 3, 7, and 14 days, which confirmed the osteoblast phenotype (Fig. 1C). Next, the expression of SNHG1 and KLF2 were examined at different time points during osteoblast differentiation. As shown in Fig. 1D and E, the results suggested a gradual 


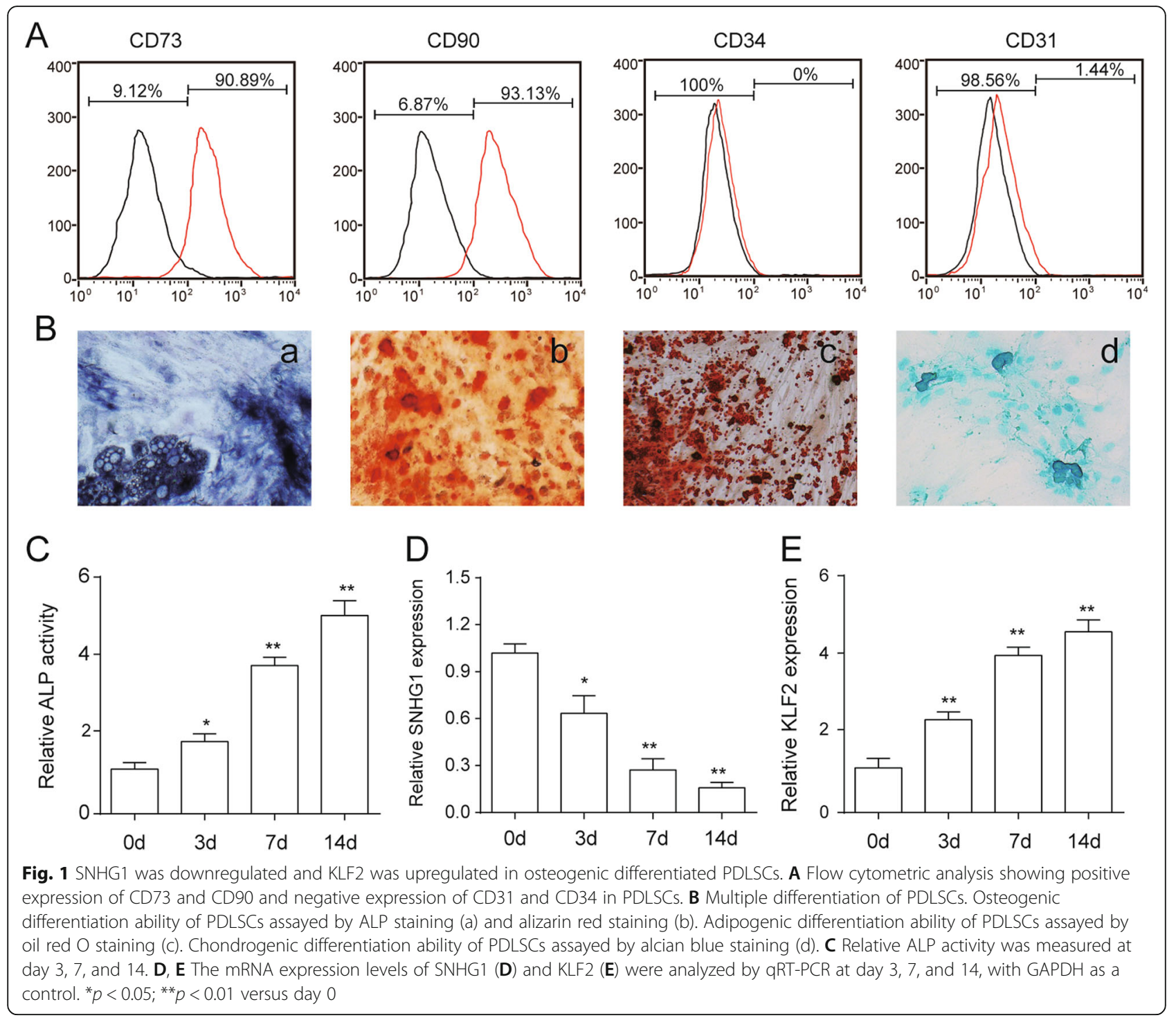

upregulation of the KLF2 expression and a gradual downregulation of the SNHG1 expression during the osteogenic induction differentiation of PDLSCs, indicating that SNHG1 and KLF2 might be involved in regulating the osteogenic differentiation of PDLSCs.

\section{SNHG1 inhibited osteogenic differentiation of PDLSCs}

To identify the biological role of SNHG1 in the regulation of PDLSCs differentiation, cells were infected with SNHG1-lentivirus to establish stably SNHG1overexpressing PDLSCs (Fig. 2a). SNHG1 overexpression apparently inhibited osteogenic differentiation, which was indicated by the downregulated osteogenic marker genes osterix (Osx), osteocalcin (OCN), and ALP both protein and mRNA levels after OM treatment (Fig. 2b, c). In addition, KLF2 mRNA and protein expression were found to be significantly downregulated after
SNHG1 overexpression with or without OM treatment (Fig. 2b, c). Furthermore, upregulation of SNHG1 suppressed OM induced ALP activity and the matrix mineralization level (Fig. 2d, e), which confirmed that SNHG1 plays a negative role in the regulation of the osteogenic differentiation of PDLSCs.

\section{Downregulation of KLF2 inhibited osteogenic differentiation of PDLSCs}

To explore the role of KLF2 on PDLSCs osteogenic differentiation, specific shRNAs were employed to reduce KLF2 expression and sh-KLF2\#1 was selected to further study for its higher efficiency (Fig. 3a). KLF2 knockdown apparently inhibited Osx, OCN, and ALP expression after OM treatment (Fig. 3b, c). ALP staining and ALP activity assays suggested that KLF2 knockdown inhibited ALP activity which was notably enhanced after OM 


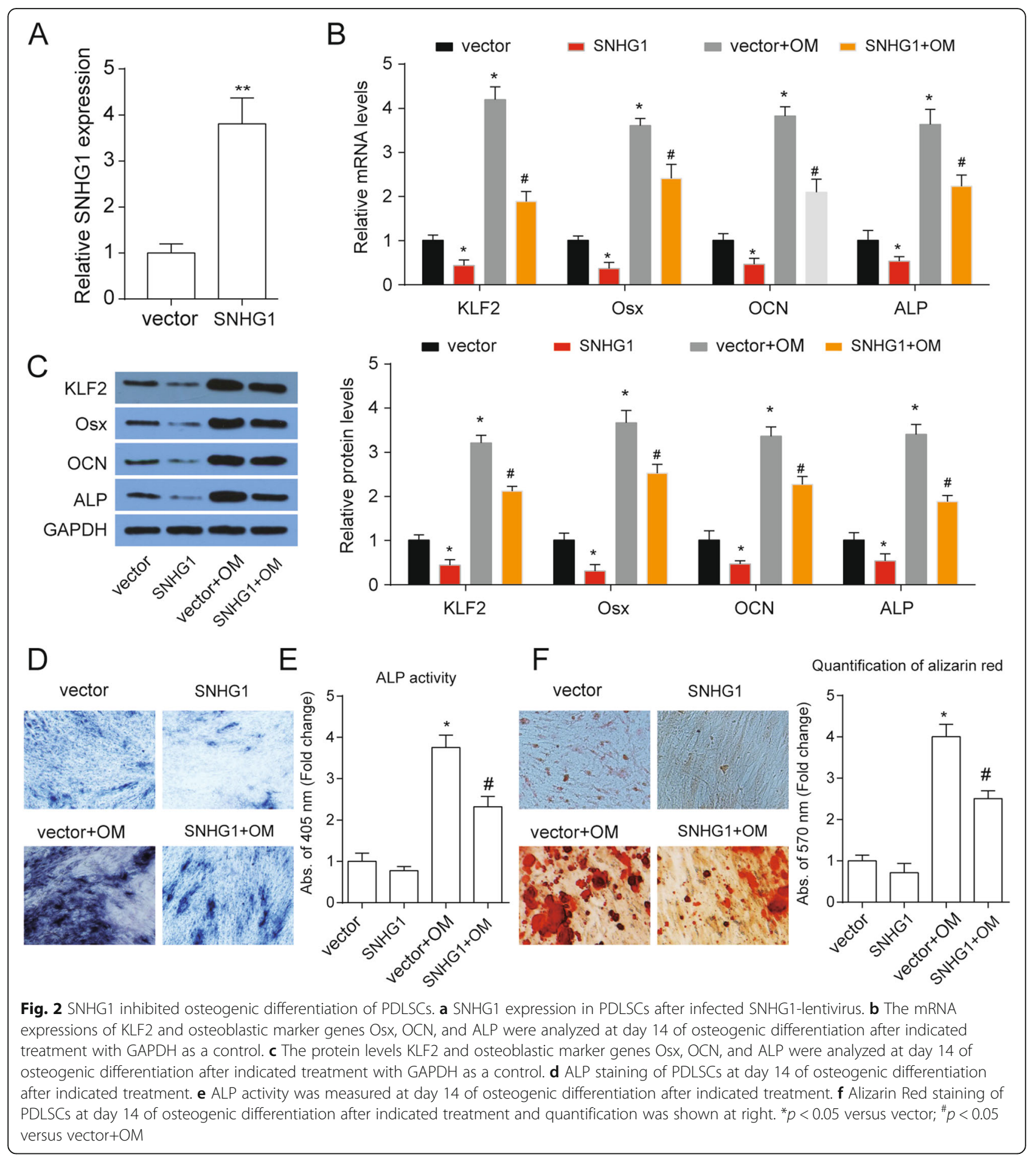

treatment (Fig. 3d, e). In addition, Alizarin Red staining confirmed the inhibitory effect of sh-KLF2 on the osteoblast phenotype with the decrease of matrix mineralization level after sh-KLF2 treatment (Fig. 3f). Taken together, these results indicated that KLF2 plays a positive role in the regulation of the osteogenic differentiation of PDLSCs.

\section{KLF2 mediates SNHG1-regulated osteogenic differentiation of PDLSCs}

To ascertain the exact role of KLF2 in SNHG1-regulated osteogenic differentiation, PDLSCs were infected with SNHG1-lentivirus or KLF2-lentivirus. As shown in Fig. 4a, SNHG1 inhibited KLF2 expression and overexpression of KLF2 recovered KLF2 expression decreased 


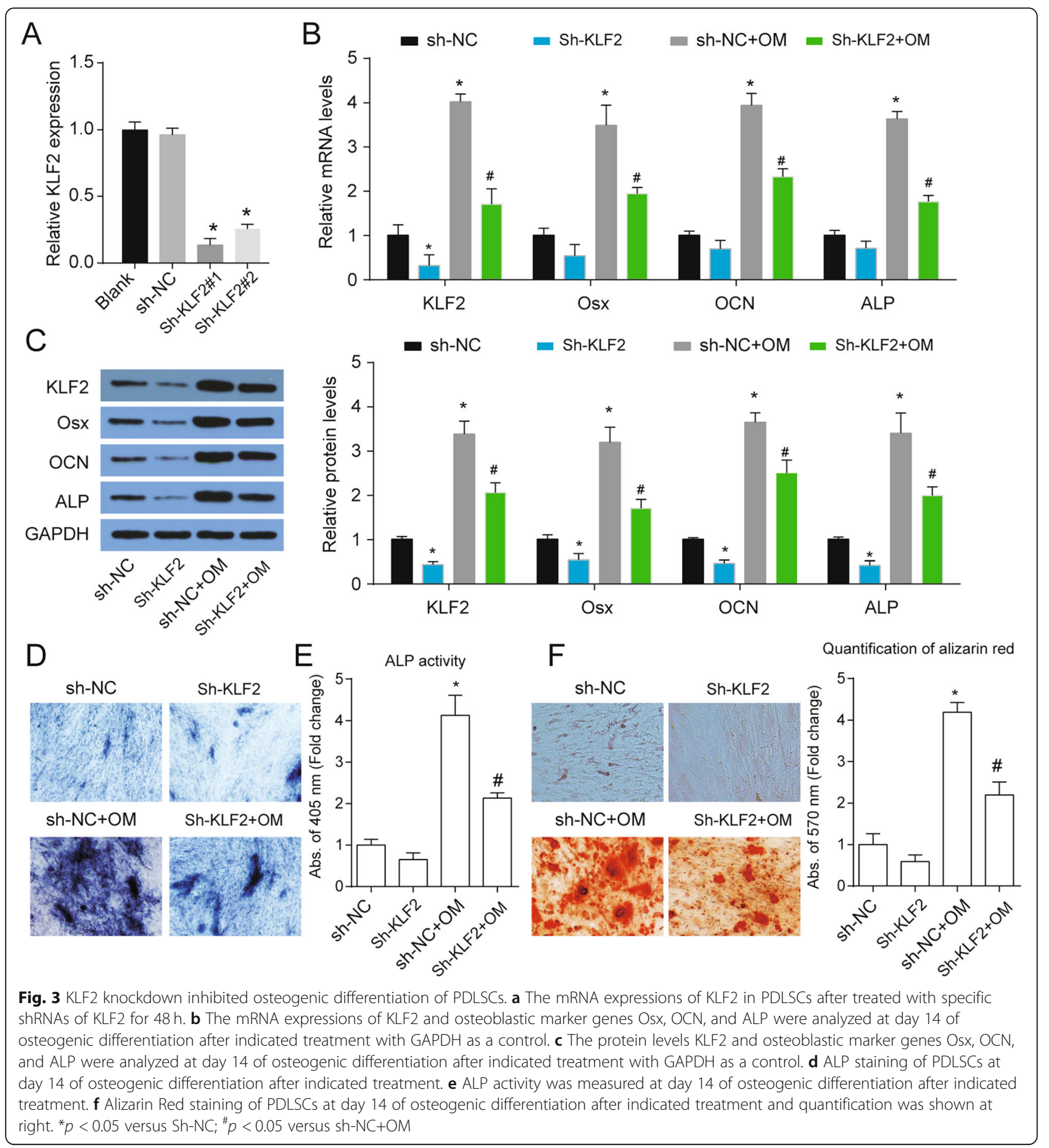

by SNHG1. KLF2 overexpression did not affect SNHG1 levels. What is more, SNHG1 overexpression inhibited osteogenic differentiation of PDLSCs as the downregulated of osteogenic marker genes Osx, OCN, and ALP and decrease of ALP activity and the matrix mineralization level (Fig. 4b-f). KLF2 overexpression promoted osteogenic differentiation of PDLSCs and KLF2 overexpression impaired the effect of SNHG1 in the osteogenic differentiation of PDLSCs (Fig. 4b-f). These results indicated that KLF2 was involved in SNHG1-regulated osteogenic differentiation of PDLSCs.

\section{SNHG1 participates in epigenetic repression of KLF2 by interacting with EZH2}

There are reports that a number of lncRNAs have been validated for working through the co-operation with 


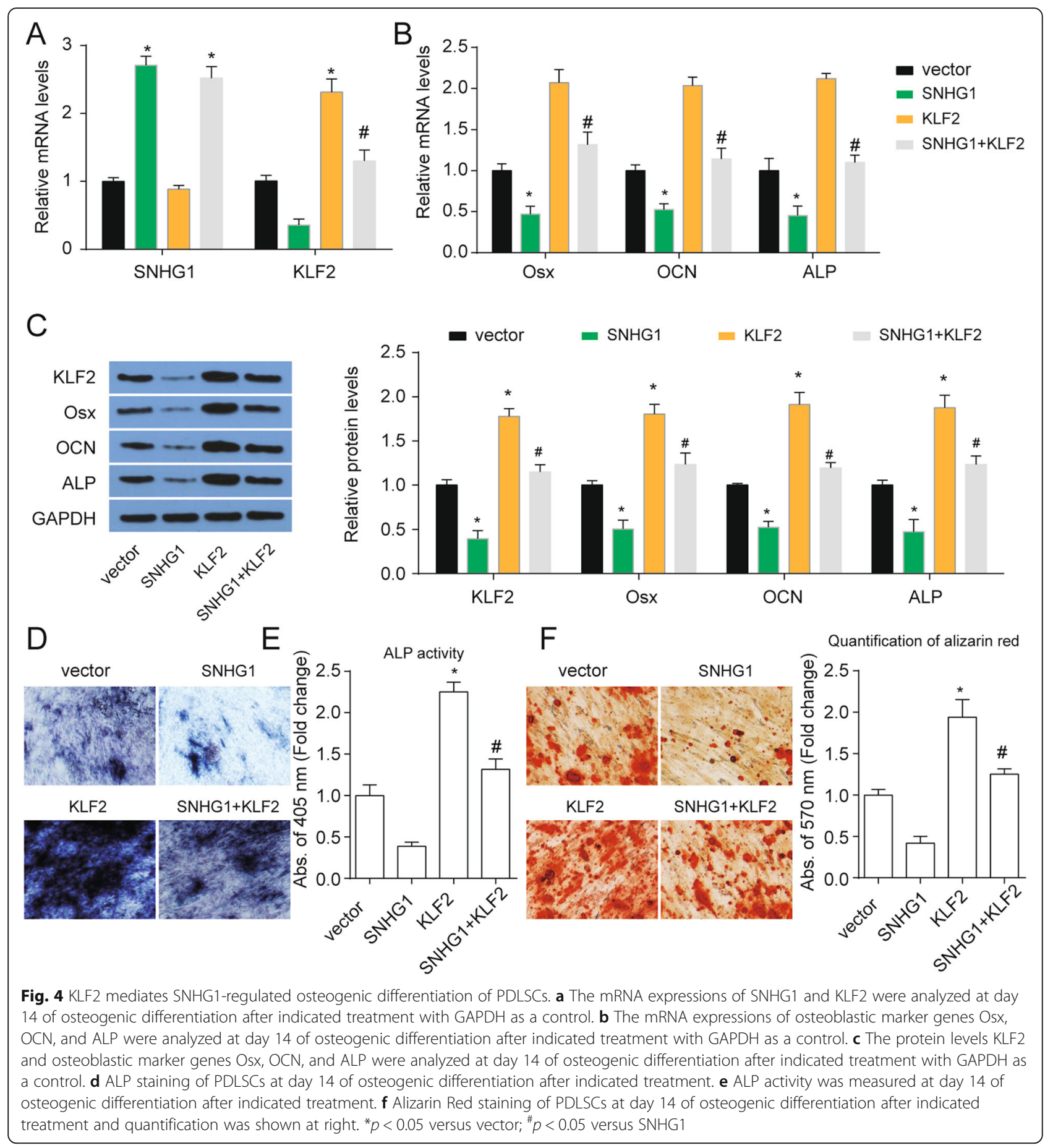

chromatin-modifying enzymes and then accelerate epigenetic activation and silence the target gene expression [25]. As evident from Fig. 5a, amplification of endogenous SNHG1 was observed in the anti-EZH2 RNA immunoprecipitation fraction associated with the input, when compared with the IgG fraction in the PDLSCs with or without OM treatment. Importantly, the levels of endogenous SNHG1 was higher in osteogenic PDLSCs compared with non-induced PDLSCs. SNHG1 overexpression increased H3K27me3 protein levels suggesting that the total methylation levels of PDLSCs were enhanced after SNHG1 overexpression (Fig. 5b). We also found that SNHG1 overexpression did not affect EZH2 expression. Downregulation of EZH2 obviously inhibited EZH2 and H3K27me3 protein levels. Sh-EZH2 rescued EZH2 and H3K27me3 protein levels enhanced by 


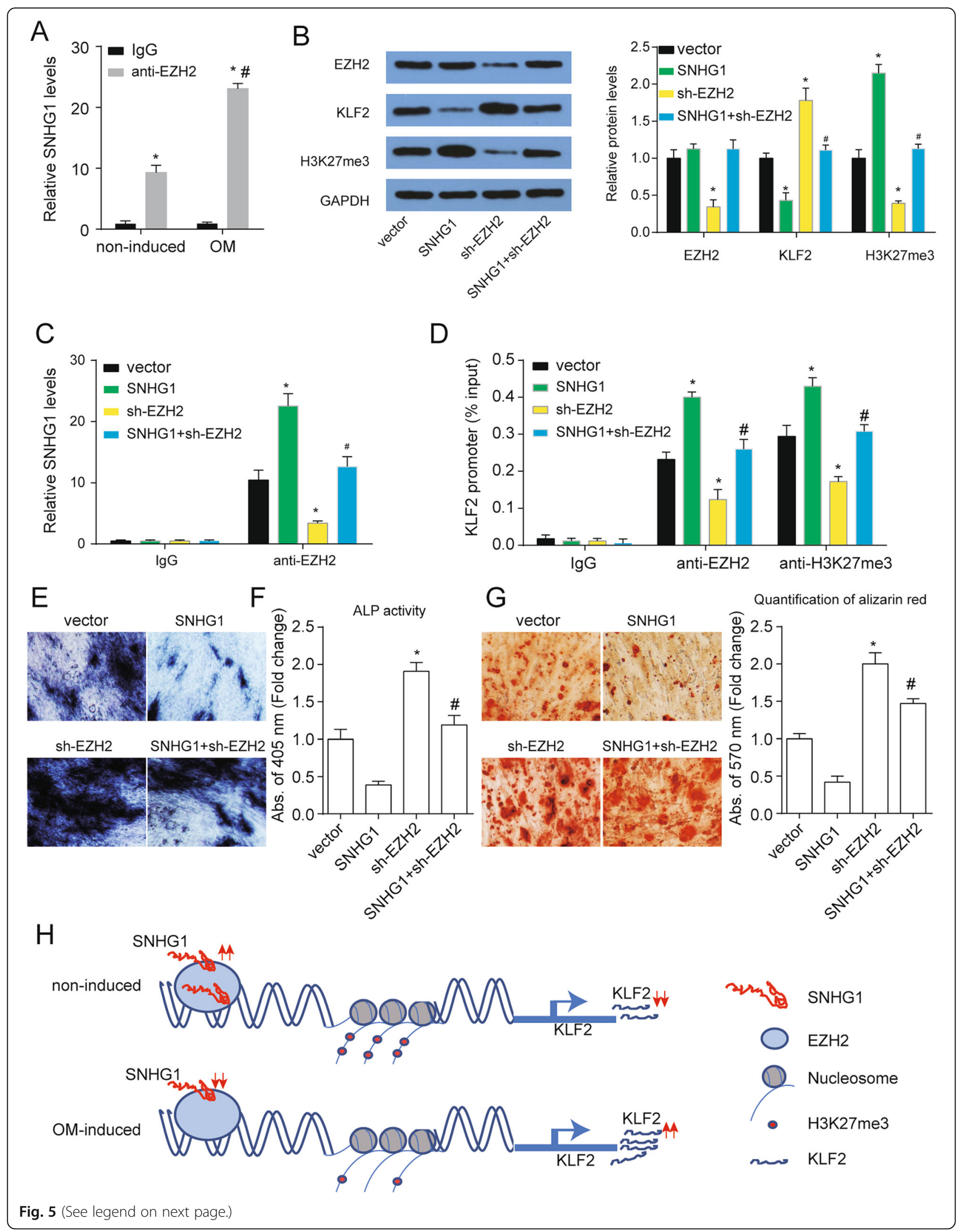


(See figure on previous page.)

Fig. 5 SNHG1 binds with EZH2 leading to epigenetically silencing of KLF2. a RIPs experiments for EZH2 were performed and the coprecipitated RNA was subjected to qRT-PCR for SNHG1 in PDLSCs with or without OM treatment. ${ }^{*} p<0.05$ versus IgG; ${ }^{*} p<0.05$ versus non-induced. $\mathbf{b}$ The protein levels EZH2, KLF2, and H3K27me3 were analyzed at day 14 of osteogenic differentiation after indicated treatment with GAPDH as a control. c RIPs experiments for EZH2 were performed and the coprecipitated RNA was subjected to qRT-PCR for SNHG1 in PDLSCs after indicated treatment along with OM treatment. $\mathbf{d}$ ChIP assays were performed to detect EZH2 and H3K27me3 occupancy in the KLF2 promoter region. e ALP staining of PDLSCs at day 14 of osteogenic differentiation after indicated treatment. $\mathbf{f}$ ALP activity was measured at day 14 of osteogenic differentiation after indicated treatment. $\mathbf{g}$ Alizarin Red staining of PDLSCs at day 14 of osteogenic differentiation after indicated treatment and quantification was shown at right. $\mathbf{h}$ Proposed model of SNHG1 regulating KLF2 expression to inhibit osteogenic differentiation of PDLSCs. ${ }^{*} p<$ 0.05 versus vector; ${ }^{\#} p<0.05$ versus SNHG1

SNHG1 overexpression (Fig. 5b). In addition, Downregulation of EZH2 promoted SLF2 expression and rescued SLF2 protein level inhibited by SNHG1 (Fig. 5b). SNHG1 overexpression enhanced the enrichment of SNHG1 in product pulled-down by EZHE antibody and sh-EZH2 treatment decreased the enrichment of SNHG1 in product pulled-down by EZHE antibody (Fig. 5c). To further address whether SNHG1 was involved in transcriptional repression through enrichment of H3K27me3 to the promoter regions of KLF2, we conducted ChIP assays. We found that SNHG1 overexpression increased EZH2 binding ability to their promoters. Similarly, EZH2 enrichment induced H3K27me3 modifications also increased in their promoter regions after SNHG1 overexpression (Fig. 5d). Down-expression of EZH2 rescued the enrichment of EZH2 and H3K27me3 on the promoter regions of KLF2 induced by SNHG1. SNHG1 knockdown notably inhibited SNHG1 expression and promoted KLF2 expression, whereas there was no significant change for EZH2 expression (Figure S1A). In addition, SNHG1 knockdown reduced the enrichment for EZH2 and H3K27me3 on KLF2 promoter region (Figure S1B). Furthermore, EZH2 knockdown promoted osteogenic differentiation of PDLSCs and rescued the effect of SNHG1 in the osteogenic differentiation of PDLS Cs (Fig. 5e-g). Taken together, these results indicated that SNHG1 epigenetically silenced KLF2 transcription through EZH2-mediated H3K27me3 methylation in the progress osteogenic differentiation of PDLSCs (Fig. 5h).

\section{SNHG1/EZH2/KLF2 axis promoted PDLSC proliferation}

As cell proliferation has a strong influence on cell differentiation [26], we further explored the effect of SNHG1/ EZH2/KLF2 axis on PDLSC proliferation. SNHG1 overexpression promoted PDLSC proliferation (Figure S1A). KLF2 overexpression inhibited PDLSC proliferation, whereas KLF2 downregulation promoted PDLSC proliferation (Figure S1B-C). EZH2 downregulation inhibited PDLSC proliferation (Figure S1D). KLF2 overexpression or EZH2 downregulation partly reversed the promotion of SNHG1 on PDLSC proliferation (Figure S1C-D). In short SNHG1/EZH2/KLF2 axis promoted PDLSC proliferation.

\section{Discussion}

Osteogenic differentiation is one of the most crucial characteristics of the oral stem cells pluripotency. It plays an important role in periodontal tissue regeneration and engineering. However, the regulatory mechanisms of MSC fate determination remain poorly understood. In this study, we found that epigenetic silencing of KLF2 by lncRNA SNHG1 inhibits PDLSC osteogenesis differentiation.

LncRNAs were reported to be involved in many biological processes, such as transcription, posttranscription, and translational regulation of gene expression [27]. LncRNA may act as a competing endogenous (ceRNA) for miRNAs, adjusting the expression of their targeting genes in the osteogenic differentiation of MSC $[28,29]$. Recently reports have also demonstrated that lncRNA could serve as epigenetic regulators participating in the osteogenic differentiation of MSC. For example, lncRNA MEG3 inhibited osteogenic differentiation of human dental follicle stem cells by epigenetically regulating wnt pathway [30]. HoxA-AS3 was reported to act as an epigenetic switch through binding with EZH2 and inhibit the transcription of key osteoblastic factors in bone marrow mesenchymal stem cells [31]. HOTAIRM1 promoted osteogenesis of dental follicle stem cells by epigenetically regulating HOXA2 [32]. In our study, we found that SNHG1 epigenetically silenced KLF2 transcription through EZH2-mediated H3K27me3 methylation in the progress osteogenic differentiation of PDLSCs.

EZH2 has been reported to be involved in various biological processes including cell growth and differentiation. Recent studies unraveled an importance role of EZH2 in regulating osteogenic differentiation [33, 34]. EZH2 induced Histone H3 lysine 27 (H3K27) methylation resulting in transcriptional repression of osteoblastic factors [31]. However, the underlying mechanisms of the impact of EZH2 on PDLSCs osteogenic differentiation still need to be explored. In this study, we found the binding of EZH2 and SNHG1 enhanced in the osteogenesis of PDLSCs. Furthermore, ChIP-PCR analysis showed that inhibition of EZH2 depressed KLF2 transcription. We confirmed that SNHG1/EZH2 
regulates the osteogenesis of PDLSCs through epigenetically mediating KLF2 expression.

Krüppel-like factor 2 (KLF2) is a zinc finger structure and DNA-binding transcription factor and is reported to possesses multiple biological functions as cellular growth and differentiation. It has been reported that KLF2 regulates cellular growth and differentiation [35]. KLF2 was involved in degradation of type II collagen, a primary component of the extracellular matrix in articular cartilage, through inhibiting matrix metalloproteinase-13 expression [36]. Interestingly, KLF2 was reported to be significantly increased during the osteoblastic differentiation process and KLF2 could promote the expression of the osteoblastic differentiation marker genes Alp, Osx, and Ocn and stimulate mineralization by enhancing the expression of and interacting with Runx2 [37]. In our study, we also found that the expression of KLF2 was upregulated during the osteoblastic differentiation process of PDLSCs and KLF2 knockdown could inhibit the expression of the osteoblastic differentiation marker genes Alp, Osx, and Ocn and stimulate mineralization.

\section{Conclusions}

SNHG1 inhibited the osteogenic differentiation of PDLS Cs through EZH2-mediated H3K27me3 methylation of KLF2 promotor. Our results provide new insight indicating that SNHG1-KLF2 axis possesses great potential as a novel class of therapeutic targets for bone regeneration.

\section{Supplementary information}

Supplementary information accompanies this paper at https://doi.org/10. 1186/s13287-020-01953-8.

Additional file 1: Supplementary table 1. The sequences of shRNA. Additional file 2: Supplementary Table 2. The primers used in this manuscript.

Additional file 3: Supplementary Table 3. The antibody used in this manuscript.

Additional file 4: Supplementary Figure 1. (A) The expression of SNHG1, EZH2 and KLF2 after SNHG1 knockdown. (B) The enrichment for EZH2 and H3K27me3 on KLF2 promoter region after SNHG1 knockdown. ${ }^{*} p<0.05$ vs. sh-NC. (C) The cell viability of PDLSCs after SNHG1 overexpression. ${ }^{* *} p<0.01$ vs. vector. (D) The cell viability of PDLSCs after KLF2 knockdown. ${ }^{* *} p<0.01$ vs. sh-NC. (E) The cell viability of PDLSCs after altering SNHG1 or KLF2 expression. ${ }^{* *} p<0.01$ vs. vector. (F) The cell viability of PDLSCs after altering SNHG1 or EZH2 expression. ${ }^{*} p<0.05$ vs. vector.

\footnotetext{
Abbreviations

ANOVA: Analysis of variance; ChIP: Chromatin immunoprecipitation; ECL: Enhanced chemiluminescence; EZH2: Enhancer of the zeste homolog 2; H3K27: Histone H3 lysine 27; KLF2: Kruppel-like factor 2; IncRNAs: Long noncoding RNAs; OM: Osteogenic differentiation medium; PDL: Periodontal ligament; PDLSCs: Periodontal ligament stem cells
}

\section{Authors' contributions}

Zhaobao Li and Xiangjun Guo have given substantial contributions to the conception or the design of the manuscript, and Zhaobao Li and Xiangjun Guo to acquisition, analysis, and interpretation of the data. All authors have participated to drafting the manuscript, and Shuainan Wu revised it critically. All authors read and approved the final version of the manuscript.

Funding

Not applicable.

Availability of data and materials

The dataset(s) supporting the conclusions of this article is (are) included within the article.

Ethics approval and consent to participate

The study protocol was approved by the Cangzhou Central Hospital on human research.

\section{Consent for publication}

All patients have provided written informed consent for the publication of any associated data and accompanying images.

\section{Competing interests}

The authors declare that they have no competing interests.

Received: 4 July 2020 Accepted: 25 September 2020

Published online: 07 October 2020

\section{References}

1. Liu Y, Zheng Y, Ding G, Fang D, Zhang C, Bartold PM, et al. Periodontal ligament stem cell-mediated treatment for periodontitis in miniature swine. Stem Cells. 2008;26:1065-73.

2. Aly LA. Stem cells: sources, and regenerative therapies in dental research and practice. World J Stem Cells. 2015;7:1047-53.

3. Lee JH, Pryce BA, Schweitzer R, Ryder MI, Ho SP. Differentiating zones at periodontal ligament-bone and periodontal ligament-cementum entheses. J Periodontal Res. 2015;50:870-80.

4. Chen FM, Sun HH, Lu H, Yu Q. Stem cell-delivery therapeutics for periodontal tissue regeneration. Biomaterials. 2012;33:6320-44.

5. Chen B, Dragomir MP, Fabris L, Bayraktar R, Knutsen E, Liu X, et al. The long noncoding RNA CCAT2 induces chromosomal instability through BOP1 AURKB signaling. Gastroenterology. 2020;14:S0016-5085(20)35057-5.

6. Tu C, Yang K, Wan L, He J, Qi L, Wang W, et al. The crosstalk between IncRNAs and the Hippo signalling pathway in cancer progression. Cell Prolif. 2020;53:e12887

7. Zhou C, Yi C, Yi Y, Qin W, Yan Y, Dong X, et al. LncRNA PVT1 promotes gemcitabine resistance of pancreatic cancer via activating Wnt/beta-catenin and autophagy pathway through modulating the miR-619-5p/Pygo2 and miR-619-5p/ATG14 axes. Mol Cancer. 2020;19:118.

8. Sayad A, Mirzajani S, Gholami L, Razzaghi P, Ghafouri-Fard S, Taheri M. Emerging role of long non-coding RNAs in the pathogenesis of periodontitis. Biomed Pharmacother. 2020;129:110362.

9. Sayad A, Ghafouri-Fard S, Shams B, Arsang-Jang S, Gholami L, Taheri M. Blood and tissue levels of IncRNAs in periodontitis. J Cell Physiol. 2020;235: 9568-76.

10. Marques-Rocha JL, Samblas M, Milagro Fl, Bressan J, Martinez JA, Marti A. Noncoding RNAs, cytokines, and inflammation-related diseases. FASEB J. 2015;29:3595-611.

11. Peng DW, Zhang J, Pei G, Rong Q, Zhu S. Long noncoding RNA ANCR suppresses bone formation of periodontal ligament stem cells via sponging miRNA-758. Biochem Biophys Res Commun. 2018;503:815-21.

12. Gong YY, Peng MY, Yin DQ, Yang YF. Long non-coding RNA H19 promotes the osteogenic differentiation of rat ectomesenchymal stem cells via Wnt/beta-catenin signaling pathway. Eur Rev Med Pharmacol Sci. 2018;22:8805-13.

13. Jiang $Y$, Wu W, Jiao G, Chen $Y$, Liu H. LncRNA SNHG1 modulates p38 MAPK pathway through Nedd4 and thus inhibits osteogenic differentiation of bone marrow mesenchymal stem cells. Life Sci. 2019;228:208-14.

14. Lim LJ, Wong SYS, Huang F, Lim S, Chong SS, Ooi LL, et al. Roles and regulation of long noncoding RNAs in hepatocellular carcinoma. Cancer Res. 2019;79:5131-9. 
15. Ou M, Li X, Zhao S, Cui S, Tu J. Long non-coding RNA CDKN2B-AS1 contributes to atherosclerotic plaque formation by forming RNA-DNA triplex in the CDKN2B promoter. EBioMedicine. 2020;55:102694.

16. Lv L, He L, Chen S, Yu Y, Che G, Tao X, et al. Long non-coding RNA LINC00114 facilitates colorectal cancer development through EZH2/DNMT1induced miR-133b suppression. Front Oncol. 2019;9:1383.

17. Su M, Xiao Y, Tang J, Wu J, Ma J, Tian B, et al. Role of IncRNA and EZH2 interaction/regulatory network in lung cancer. J Cancer. 2018;9:4156-65.

18. Wang Q, Liu L, Zhang S, Ming Y, Liu S, Cheng K, et al. Long noncoding RNA NEAT1 suppresses hepatocyte proliferation in fulminant hepatic failure through increased recruitment of EZH2 to the LATS2 promoter region and promotion of H3K27me3 methylation. Exp Mol Med. 2020;52:461-72.

19. Yu Y, Zhang M, Wang N, Li Q, Yang J, Yan S, et al. Epigenetic silencing of tumor suppressor gene CDKN1A by oncogenic long non-coding RNA SNHG1 in cholangiocarcinoma. Cell Death Dis. 2018:9:746.

20. Xu M, Chen X, Lin K, Zeng K, Liu X, Pan B, et al. The long noncoding RNA SNHG1 regulates colorectal cancer cell growth through interactions with EZH2 and miR-154-5p. Mol Cancer. 2018;17:141.

21. Seo BM, Miura M, Gronthos S, Bartold PM, Batouli S, Brahim J, et al. Investigation of multipotent postnatal stem cells from human periodontal ligament. Lancet. 2004;364:149-55.

22. Liu Y, Yang H, Wen Y, Li B, Zhao Y, Xing J, et al. Nrf2 inhibits periodontal ligament stem cell apoptosis under excessive oxidative stress. Int J Mol Sci. 2017;18:1076.

23. Wen $Y$, Yang H, Wu J, Wang A, Chen X, Hu S, et al. COL4A2 in the tissuespecific extracellular matrix plays important role on osteogenic differentiation of periodontal ligament stem cells. Theranostics. 2019;9:4265-86.

24. Liu D, Wang Y, Jia Z, Wang L, Wang J, Yang D, et al. Demethylation of IGFBP5 by histone demethylase KDM6B promotes mesenchymal stem cell-mediated periodontal tissue regeneration by enhancing osteogenic differentiation and anti-inflammation potentials. Stem Cells. 2015;33:2523-36.

25. Marchese FP, Huarte M. Long non-coding RNAs and chromatin modifiers: their place in the epigenetic code. Epigenetics. 2014;9:21-6.

26. Fernandez-Barral A, Bustamante-Madrid P, Ferrer-Mayorga G, Barbachano A, Larriba MJ, Munoz A. Vitamin D effects on cell differentiation and stemness in cancer. Cancers (Basel). 2020;12:E2413.

27. Quinn JJ, Chang HY. Unique features of long non-coding RNA biogenesis and function. Nat Rev Genet. 2016;17:47-62.

28. Wang L, Wu F, Song Y, Li X, Wu Q, Duan Y, et al. Long noncoding RNA related to periodontitis interacts with miR-182 to upregulate osteogenic differentiation in periodontal mesenchymal stem cells of periodontitis patients. Cell Death Dis. 2016;7:e2327.

29. Liang WC, Fu WM, Wang YB, Sun YX, Xu LL, Wong CW, et al. H19 activates Wnt signaling and promotes osteoblast differentiation by functioning as a competing endogenous RNA. Sci Rep. 2016;6:20121.

30. Deng L, Hong H, Zhang X, Chen D, Chen Z, Ling J, et al. Down-regulated IncRNA MEG3 promotes osteogenic differentiation of human dental follicle stem cells by epigenetically regulating Wnt pathway. Biochem Biophys Res Commun. 2018;503:2061-7.

31. Zhu XX, Yan YW, Chen D, Ai CZ, Lu X, Xu SS, et al. Long non-coding RNA HoxA-AS3 interacts with EZH2 to regulate lineage commitment of mesenchymal stem cells. Oncotarget. 2016;7:63561-70.

32. Chen Z, Zheng J, Hong H, Chen D, Deng L, Zhang X, et al. IncRNA HOTAIRM1 promotes osteogenesis of hDFSCs by epigenetically regulating HOXA2 via DNMT1 in vitro. J Cell Physiol. 2020;235:8507-19.

33. Hemming S, Cakouros D, Vandyke K, Davis MJ, Zannettino AC, Gronthos S. Identification of novel EZH2 targets regulating osteogenic differentiation in mesenchymal stem cells. Stem Cells Dev. 2016;25:909-21.

34. Dudakovic A, Samsonraj RM, Paradise CR, Galeano-Garces C, Mol MO, GaleanoGarces D, et al. Inhibition of the epigenetic suppressor EZH2 primes osteogenic differentiation mediated by BMP2. J Biol Chem. 2020;295:7877-93.

35. McConnell BB, Yang WW. Mammalian Kruppel-like factors in health and diseases. Physiol Rev. 2010;90:1337-81.

36. Yuan Y, Tan H, Dai P. Kruppel-like factor 2 regulates degradation of type II collagen by suppressing the expression of matrix metalloproteinase (MMP)13. Cell Physiol Biochem. 2017:42:2159-68.

37. Hou Z, Wang Z, Tao Y, Bai J, Yu B, Shen J, et al. KLF2 regulates osteoblast differentiation by targeting of Runx2. Lab Investig. 2019;99:271-80.

\section{Publisher's Note}

Springer Nature remains neutral with regard to jurisdictional claims in published maps and institutional affiliations.

\section{Ready to submit your research? Choose BMC and benefit from}

- fast, convenient online submission

- thorough peer review by experienced researchers in your field

- rapid publication on acceptance

- support for research data, including large and complex data types

- gold Open Access which fosters wider collaboration and increased citations

- maximum visibility for your research: over $100 \mathrm{M}$ website views per year

At BMC, research is always in progress.

Learn more biomedcentral.com/submissions 